\title{
Absorption of energy at a metallic surface due to a normal electric field
}

\author{
Michael Wilkinson \\ Faculty of Mathematics and Computing, Open University, Walton Hall, \\ Milton Keynes MK7 6AA, UK
}

Received 13 November 2000, in final form 17 January 2001

\begin{abstract}
The effect of an oscillating electric field normal to a metallic surface may be described by an effective potential. This induced potential is calculated using semiclassical variants of the random-phase approximation. Results are obtained for both ballistic and diffusive electron motion, and for two- and three-dimensional systems. The potential induced within the surface causes absorption of energy. The results are applied to the absorption of radiation by small metal spheres and discs. They improve upon an earlier treatment which used the Thomas-Fermi approximation for the effective potential.
\end{abstract}

\section{Introduction}

When an external electric field is applied to a metal, polarization charges are induced on the surface. At zero frequency, these charges create an additional potential which exactly cancels the applied electric field within the interior of the metal. This paper discusses the form of the potential at frequencies $\omega$ which are small compared to the plasma frequency of the metal, $\omega_{\mathrm{p}}$, for the case where the external field is perpendicular to the surface of the metal. The potential is calculated self-consistently, using two distinct simplified versions of the 'random-phase approximation' (RPA) approach. The standard random-phase approximation is discussed in [1]. The formulation of the 'semiclassical' variants of the RPA used in this paper was discussed in [2].

This effective potential is not directly measurable, but it does have an influence on the electromagnetic response of the surface, in particular on the absorption of radiation. The results will be used to address a long-standing problem, concerning the theory for absorption of radiation by small conducting particles. Standard electromagnetic theory predicts that the absorption coefficient $\alpha(\omega)$ of a dispersion of small metal particles is proportional to $\omega^{2}$, and the coefficients are known for simple geometries such as spheres [3], or discs with the electric field vector in the plane of the disc [4]. In the standard treatment the conductivity of the metal is assumed to be local, with the current density at position $r$ proportional to the electric field at $\boldsymbol{r}$. This assumption is valid if the motion of electrons is diffusive. In very small particles the bulk mean free path may be larger than the size of the particle, and in this ballistic case the conductivity must be treated as a non-local quantity. The frequency dependence of the 
absorption coefficient has not received a satisfactory analytical treatment in the ballistic case. The final result of this paper will be estimates for the absorption coefficient of the form

$$
\begin{aligned}
\alpha(\omega) & \sim K_{3} \omega^{2} & & \text { spheres } \\
\alpha(\omega) & \sim K_{2} \omega & & \text { discs. }
\end{aligned}
$$

In the latter case, the electric field is polarized in the plane of the disc.

There is a large literature concerned with numerical approaches to calculating the absorption coefficient. A notable contribution was the implementation of the RPA equations for ballistic electrons in a spherical geometry, described in reference [5] (which also gives a bibliography of earlier numerical work). The numerical calculations capture many effects which are ignored in this analytical treatment, such as absorption resonances, quantum size effects, and shell effects. It is however surprising that while there is an understanding of many of these features, there has been no satisfactory theory describing the background growth of the absorption, on which these spectral features are superposed. The calculation in this paper should be seen as complementing the numerical approach, providing physical insight and a benchmark analytical formula against which numerical calculations can be compared.

In two earlier papers I discussed the absorption of low-frequency radiation in particles with ballistic electron motion using a Thomas-Fermi approximation: reference [6] treated spherical particles in three dimensions, and reference [7] discussed discs with the electric field polarized in the conducting plane. The surface may be smooth enough that the electron is reflected specularly, in which case the motion is integrable due to conservation of angular momentum, or it may be a rough surface resulting in ergodic electron motion. The earlier works [6] and [7] consider both integrable and ergodic cases. It was shown that in the integrable case the absorption coefficient is the sum of contributions from resonant absorption by electrons with angles of incidence $\theta$ satisfying the condition

$$
\left(v_{\mathrm{F}} / a \omega\right)\left(n \pi \pm \theta_{n}\right)=\sin \theta_{n}
$$

where the integer $n$ labels the resonance, $v_{\mathrm{F}}$ is the Fermi velocity, and $a$ is the radius. There are no resonances below the frequency $\omega_{\mathrm{c}}=v_{\mathrm{F}} / a$, so there is no absorption when $\omega<\omega_{\mathrm{c}}$. As the frequency increases, more resonances contribute, and it was shown that when $\omega \gg \omega_{\mathrm{c}}$, the frequency-averaged behaviour of the sum of these resonances is of the form (1.1). In the case of ergodic electron motion there are no resonances, but (1.1) continues to apply (although the coefficients $K_{2}$ and $K_{3}$ are different). The prediction that the absorption coefficient is proportional to frequency in the two-dimensional case was a surprising result.

In [6] and [7] it was also shown that when $\omega \gg \omega_{\mathrm{c}}$, the frequency-averaged absorption can be obtained correctly by treating the collisions of the electron with the surface as if they are independent events. The present paper adopts this simplification, which avoids summing over contributions from a large number of resonances.

Reference [2] gave a comprehensive discussion of the equations underlying the treatment of absorption of radiation, and concluded that the Thomas-Fermi approximation is not sufficient, even when the electron motion is ballistic. The form of the potential was shown to be very different from the Thomas-Fermi approximation when $\omega \gg \omega_{\mathrm{c}}$. The conclusions of references [6] and [7] should therefore be re-evaluated, particularly the prediction that $\alpha(\omega) \sim \omega$ in the two-dimensional case. This paper treats absorption by particles with ballistic electron motion using two distinct approximations to the 'random-phase approximation', instead of the simpler Thomas-Fermi approximation used in [6] and [7]. The conclusions are consistent with (1.1) (but different values for the coefficients $K_{3}$ and $K_{2}$ are obtained).

Two simplifications of the RPA approach are discussed in section 2 . The first will be termed the 'image source approximation', and leads to a slight simplification of the RPA equations. 
It is valid in the neighbourhood of a flat surface, when the frequency is small compared to the plasma frequency. The second approach, previously discussed in [2], will be termed the 'semiclassical' RPA method. It makes more radical assumptions, and leads to simpler equations. The results of both approaches are approximate, rather than being the leading term of an asymptotic theory. Section 3 obtains the self-consistent effective potential which describes the electric field within the surface. First this is calculated using the semiclassical RPA method for both two- and three-dimensional systems, and then using the image source approximation for the simpler case of a three-dimensional system. Section 4 calculates $\delta E(\theta)$, the transfer of energy to an electron colliding with the surface, as a function of the angle of incidence $\theta$. Again, the calculation is carried out first for the semiclassical RPA method, and then using the image source approximation in the three-dimensional case.

When electrons collide with the boundary, $\delta E$ may be either positive or negative, depending on the phase of the oscillating electric field. The energy transfer may be treated as a random variable, so that the evolution of the energy of an electron over many collisions is diffusive. Absorption of energy results from the diffusive excitation of electrons in filled states below the Fermi level, into empty states at higher energies. The absorption coefficient is calculated in section 5 by taking suitable averages of $\delta E^{2}(\theta)$, for both spheres and discs. The calculation is carried out for both ergodic and integrable electron motion, resulting in different numerical coefficients in the expressions for $K_{2}$ and $K_{3}$. Section 6 is a brief discussion of the validity of the results.

This work uses a free-electron model for the conduction electrons, with an infinite potential outside the surface, as described in [6]. The symbol $e$ will denote the magnitude of the electron charge, and the potential $\phi$ will denote the potential energy of an electron (rather than the electrostatic potential). Following common practice, equations will be written as equalities, despite the fact that most of them are approximate relations. References [2] and [6] contain ample discussion of and references to the existing literature.

\section{Two semiclassical RPA methods}

\subsection{The RPA equations}

A polarizable medium is perturbed by applying a time-dependent external potential, which is specified in the frequency domain by a function $\phi_{\text {ext }}(r, \omega)$. The motion of electrons within the medium may be approximated by an independent-particle effective Hamiltonian, which contains an effective potential $\phi(r, \omega)$. By analogy with Dirac notation, these potentials can be denoted by function-space vectors, $\left.\mid \phi_{\text {ext }}\right)$ and $\left.\mid \phi\right)$ respectively, and I shall write $\phi(r, \omega)=(\boldsymbol{r} \mid \phi)$. The effective potential must take account of the fact that the medium is polarized by the externally applied field, so the effective potential is the sum of the external potential and the potential $\left.\mid \phi_{\text {pol }}\right)$ generated by the polarization charge $\left.\mid \rho\right)$ through the action of the 'Coulomb operator' $\hat{U}$, which is defined through the relations

$$
\phi_{\mathrm{pol}}(\boldsymbol{r}, \omega) \equiv\left(\boldsymbol{r} \mid \phi_{\mathrm{pol}}\right)=(\boldsymbol{r}|\hat{U}| \rho) \equiv \frac{-e}{4 \pi \epsilon_{0}} \int \mathrm{d} \boldsymbol{r}^{\prime} \frac{1}{\left|\boldsymbol{r}-\boldsymbol{r}^{\prime}\right|} \rho\left(\boldsymbol{r}^{\prime}, \omega\right) .
$$

The induced charge density $\mid \rho$ ) is obtained from the effective potential $\mid \phi$ ) by multiplication by the polarizability operator $\hat{\Pi}(\omega)$, i.e. $\mid \rho)=\hat{\Pi}(\omega) \mid \phi)$. Writing $\left.\left.\mid \phi)=\mid \phi_{\text {ext }}\right)+\mid \phi_{\text {pol }}\right)$ gives a single equation which should be solved for the effective potential: the RPA equation is

$$
\left.\left.\mid \phi)=\mid \phi_{\mathrm{ext}}\right)+\hat{U} \hat{\Pi}(\omega) \mid \phi\right)
$$

A thorough discussion of the RPA method is given in [1]. 


\subsection{The image source approximation}

The polarizability $\hat{\Pi}(\omega)$ is related to the spatial probability propagator by

$$
\hat{\Pi}(\omega)=e v[\hat{I}+\mathrm{i} \omega \hat{P}(\omega)]
$$

where $v$ is the density of states at the Fermi energy, $\left(\boldsymbol{r}|P(\omega)| \boldsymbol{r}^{\prime}\right)$ is the Fourier transform of the probability $P\left(\boldsymbol{r}, \boldsymbol{r}^{\prime}, t\right)$ of an electron initially at $\boldsymbol{r}^{\prime}$ being located at $\boldsymbol{r}$ after time $t$. A semiclassical derivation of this relation is given in [2].

In the vicinity of a flat surface, the coordinate-space representation of the polarizability may be approximated as follows:

$\Pi\left(\boldsymbol{r}, \boldsymbol{r}^{\prime}, \omega\right) \equiv\left(\boldsymbol{r}|\hat{\Pi}(\omega)| \boldsymbol{r}^{\prime}\right)=e \nu\left[\delta\left(\boldsymbol{r}-\boldsymbol{r}^{\prime}\right)+\mathrm{i} \omega P\left(\boldsymbol{r}-\boldsymbol{r}^{\prime}, \omega\right)+\mathrm{i} \omega P\left(\boldsymbol{r}-\boldsymbol{r}_{\mathrm{R}}^{\prime}, \omega\right)\right]$

where $P\left(\boldsymbol{r}-\boldsymbol{r}^{\prime}, \omega\right)$ is the Fourier transform of the free-space propagator $P\left(\boldsymbol{r}-\boldsymbol{r}^{\prime}, t\right)$, and $\boldsymbol{r}_{\mathrm{R}}$ is the reflection of the point $r$ in the plane of the boundary.

Specializing to the case where the boundary is a flat plane at $z=0$, and where the potential $\phi(z)$ depends only on the distance from the boundary, the equation $\mid \rho)=\hat{\Pi}(\omega) \mid \phi)$ may be written as

$$
\begin{aligned}
\rho(z)=e v \phi(z) & +\mathrm{i} e \nu \omega \int \mathrm{d} x^{\prime} \int \mathrm{d} y^{\prime} \int \mathrm{d} z^{\prime}\left[P\left(\left(x^{\prime}, y^{\prime}, z-z^{\prime}\right), \omega\right)+P\left(\left(x^{\prime}, y^{\prime}, z+z^{\prime}\right), \omega\right)\right] \\
& \times \phi\left(z^{\prime}\right) .
\end{aligned}
$$

The integrals will be taken to be over all space, so equation (2.5) is the sum of two convolutions. The interior of the metal is the region $z>0$, and I set $\phi(z)=0$ for $z<0$. Equation (2.5) will be termed the 'image source approximation'.

In view of the isotropy of the free-space propagator, its Fourier transform $\tilde{P}(\boldsymbol{k}, \omega)$ is a function of the magnitude $k=|k|$ of the wavevector:

$$
p(k, \omega)=\tilde{P}(\boldsymbol{k}, \omega) \equiv \int \mathrm{d} \boldsymbol{r} \exp [\mathrm{i} \boldsymbol{k} \cdot \boldsymbol{r}] P(\boldsymbol{r}, \omega) \quad k \equiv|\boldsymbol{k}| .
$$

The function $p(k, \omega)$ can be determined by Fourier transformation of a semiclassical approximation of the position representation of the free-space propagator, valid when $k \ll k_{\mathrm{F}}$. In [2] it was shown that the resulting Fourier representation of the free-space polarizability may be expressed in terms of a single scaling variable $\lambda$ :

$$
\Pi(k, \omega)=e \nu[1+\mathrm{i} \omega p(k, \omega)]=e \nu[1+g(\lambda)] .
$$

The dimensionless variable $\lambda$ takes different forms for ballistic or diffusive electron dynamics:

$$
\lambda= \begin{cases}k v_{\mathrm{F}} / \omega & \text { ballistic } \\ k \sqrt{D / \omega} & \text { diffusive }\end{cases}
$$

where $D$ is the diffusion constant. In the ballistic case the form of the function $g(k)$ depends upon the dimensionality of space. In two dimensions

$$
g_{2}(\lambda)= \begin{cases}-\left(1-\lambda^{2}\right)^{-1 / 2} & \lambda<1 \\ \mathrm{i}\left(\lambda^{2}-1\right)^{-1 / 2} & \lambda>1\end{cases}
$$

and in three dimensions

$$
g_{3}(\lambda)=-\frac{1}{2 \lambda} \log _{\mathrm{e}}\left|\frac{\lambda+1}{\lambda-1}\right|+\frac{\pi \mathrm{i}}{2 \lambda} \Theta(\lambda-1)
$$

(where $\Theta(x)$ is the Heaviside function, with increasing unit step at $x=0$ ). The limiting forms of $g(\lambda)$ for small and large argument are informative:

$$
g_{d}(\lambda)=-\left(1+\frac{1}{d} \lambda^{2}\right)+\mathrm{O}\left(\lambda^{3}\right)
$$




$$
\lim _{\lambda \rightarrow \infty}\left[\lambda g_{d}(\lambda)\right]= \begin{cases}\mathrm{i} & (d=2) \\ \frac{\pi}{2} \mathrm{i} & (d=3) .\end{cases}
$$

In both two- and three-dimensional ballistic motion, $g_{d}(\lambda)$ has an integrable divergence at $\lambda=1$. In the case of diffusive electron motion, the form of the function $g(\lambda)$ is the same in two and three dimensions:

$$
g_{\mathrm{D}}(\lambda)=\frac{-1}{1+\mathrm{i} \lambda^{2}} .
$$

\subsection{The semiclassical RPA approximation}

Formally, the effective potential may be determined simply by inverting (2.2):

$$
\left.\mid \phi)=[\hat{I}-\hat{U} \hat{\Pi}(\omega)]^{-1} \mid \phi_{\text {ext }}\right) .
$$

This approach can be implemented numerically by expanding functions in a suitable basis set and inverting matrices numerically. It is desirable to have a simpler approach which allows further analytical progress. In [2], a 'semiclassical' variant of the RPA approach was introduced. It is assumed that (provided that $\omega \ll \omega_{\mathrm{p}}$ ) the polarization charge $\mid \rho$ ) is that which would be predicted by classical electrostatic theory (the justification for this is discussed in [2]). Under this assumption, the effective potential satisfies a much simpler equation

$$
\left.\left.\mid \rho_{\mathrm{cl}}\right)=\hat{\Pi}(\omega) \mid \phi\right)
$$

where $\left.\mid \rho_{\mathrm{cl}}\right)$ is the classical charge distribution function resulting from a static external field, which is assumed to be known. The task of determining the effective potential is then reduced to the simpler task of inverting $\hat{\Pi}(\omega)$. Calculation of $\left.\mid \rho_{\mathrm{cl}}\right)$ is still a difficult problem, but solutions are obtained in various geometries in textbooks such as [3].

The form of the surface charge density is different in two and three dimensions. In three dimensions the charge density is confined to a narrow layer at the surface of the conductor, with thickness equal to the Thomas-Fermi screening length. This can be approximated by a delta-function distribution, a tiny distance $\varepsilon$ inside the surface:

$$
\rho(z)=q \delta(z-\varepsilon)
$$

where $q$ is the surface charge density induced by the externally applied fields. In (2.15) the coefficient $q$ depends upon the position $s$ on the surface of the conducting particle. In the case of a two-dimensional conductor in three-dimensional space, there is an inverse square-root divergence of the charge density at the surface of the particle:

$$
\rho(z)=\frac{C}{\sqrt{z}} .
$$

The reasons for this behaviour are discussed in [7].

\section{Calculation of the surface potential}

\subsection{Method for solving the semiclassical RPA equation}

Equation (2.5) will now be expressed in a purely one-dimensional form. To this end, define $F(z, \omega)$ as the inverse Fourier transform of $p(k, \omega)$ :

$$
p(k, \omega) \equiv \int_{-\infty}^{\infty} \mathrm{d} z \exp [\mathrm{i} k z] F(z, \omega)
$$


and note that

$$
\begin{aligned}
\int_{-\infty}^{\infty} \mathrm{d} x^{\prime} \int_{-\infty}^{\infty} & \mathrm{d} y^{\prime} \int_{-\infty}^{\infty} \mathrm{d} z^{\prime} P\left(\left(x^{\prime}, y^{\prime}, z-z^{\prime}\right) ; \omega\right) \phi\left(z^{\prime}\right) \\
& =-\frac{1}{(2 \pi)^{3}} \int \mathrm{d} \boldsymbol{r}^{\prime \prime} \phi\left(z-z^{\prime \prime}\right) \int \mathrm{d} \boldsymbol{K} \exp \left[-\mathrm{i} \boldsymbol{K} \cdot \boldsymbol{r}^{\prime \prime}\right] \tilde{P}(\boldsymbol{K} ; \omega) \\
& =-\frac{1}{2 \pi} \int_{-\infty}^{\infty} \mathrm{d} z^{\prime \prime} \phi\left(z-z^{\prime \prime}\right) \int \mathrm{d} \boldsymbol{K} \tilde{P}(\boldsymbol{K}, \omega) \delta\left(K_{x}\right) \delta\left(K_{y}\right) \exp \left[-\mathrm{i} \boldsymbol{K} \cdot \boldsymbol{r}^{\prime \prime}\right] \\
& =-\frac{1}{2 \pi} \int_{-\infty}^{\infty} \mathrm{d} z^{\prime \prime} \phi\left(z-z^{\prime \prime}\right) \int_{-\infty}^{\infty} \mathrm{d} k \exp \left[-\mathrm{i} k z^{\prime \prime}\right] p(k, \omega) \\
& =\int_{-\infty}^{\infty} \mathrm{d} z F\left(z-z^{\prime}, \omega\right) \phi\left(z^{\prime}\right)
\end{aligned}
$$

Using this result, the integrals over $x^{\prime}$ and $y^{\prime}$ in equation (2.5) may be eliminated, giving

$$
\rho(z)=e v\left[\phi(z)+\mathrm{i} \omega \int_{-\infty}^{\infty} \mathrm{d} z^{\prime}\left[F\left(z-z^{\prime}, \omega\right)+F\left(z+z^{\prime}, \omega\right)\right] \phi\left(z^{\prime}\right)\right] .
$$

Comparison of (3.3) with (3.1) shows that $F(z, \omega)$ is related to the inverse Fourier transform of the function $g(\lambda)$, introduced in $(2.7)$. In the ballistic case,

$$
F(z, \omega)=\frac{1}{2 \pi \mathrm{i} \omega} \int_{-\infty}^{\infty} \mathrm{d} k \exp [-\mathrm{i} k z] g\left(k v_{\mathrm{F}} / \omega\right) \equiv \frac{1}{\mathrm{i} v_{\mathrm{F}}} G\left(z \omega / v_{\mathrm{F}}\right)
$$

where $G$ is the inverse Fourier transform of $g$. Equation (3.3) can now be written in the scaled form

$$
\rho(z)=e v\left\{\phi(z)+\frac{1}{\Lambda} \int_{0}^{\infty} \mathrm{d} z^{\prime}\left[G\left(\frac{z-z^{\prime}}{\Lambda}\right)+G\left(\frac{z+z^{\prime}}{\Lambda}\right)\right] \phi\left(z^{\prime}\right)\right\}
$$

where the scale length $\Lambda$ is

$$
\Lambda= \begin{cases}v_{\mathrm{F}} / \omega & \text { ballistic } \\ \sqrt{D / \omega} & \text { diffusive }\end{cases}
$$

Defining a scaled distance by $x=z / \Lambda$, and a scaled charge density $f(x)$ and potential $\psi(x)$ by

$$
\rho(z)=e v f(x) \quad \phi(z)=\psi(x)
$$

equation (3.5) may be expressed in the dimensionless form

$$
f(x)=\psi(x)+\int_{0}^{\infty} \mathrm{d} x^{\prime}\left[G\left(x-x^{\prime}\right)+G\left(x+x^{\prime}\right)\right] \psi\left(x^{\prime}\right) .
$$

Now consider how to solve equation (3.8) for the scaled potential $\psi(x)$, given the scaled charge density $f(x)$. The function $f(x)$ is defined only for $x>0$. Also, the behaviour of $\psi(x)$ for $x<0$ is irrelevant to the form of $f(x)$ in the region where this is defined. We may define a symmetric extension of the function $f(x)$ :

$$
f_{\mathrm{s}}(x)=f(|x|) \text {. }
$$

Consider a function $\psi_{\mathrm{s}}(x)$ which satisfies

$$
f_{\mathrm{s}}(x)=\psi_{\mathrm{s}}(x)+\int_{-\infty}^{\infty} \mathrm{d} x^{\prime} G\left(x-x^{\prime}\right) \psi_{\mathrm{s}}\left(x^{\prime}\right)
$$


This function must be symmetric: $\psi_{\mathrm{s}}(x)=\psi_{\mathrm{s}}(-x)$. This symmetric solution satisfies the same equation as $\psi(x)$ (equation (3.8)) for $x<0$. We therefore solve the simpler equation (3.10), and drop the subscript s labelling the solution. The Fourier transform of the solution is

$$
\tilde{\psi}(k)=\frac{\tilde{f}_{\mathrm{s}}(k)}{1+g(k)} .
$$

The solution may also be expressed as a convolution:

$$
\psi(x)=\int_{-\infty}^{\infty} \mathrm{d} x^{\prime} K\left(x-x^{\prime}\right) f_{\mathrm{s}}\left(x^{\prime}\right)
$$

where $K(x)$ is the inverse Fourier transform of $(1+g(k))^{-1}$.

These expressions require the form of the function $f_{\mathrm{s}}(x)$. Referring to (2.15) (and taking $\varepsilon \rightarrow 0$ ), in three dimensions the Fourier transform of the scaled and symmetrized charge density is, in the ballistic case

$$
\tilde{f}_{\mathrm{s}}(k)=\frac{2 q \omega}{e \nu v_{\mathrm{F}}}
$$

The factor of 2 appears in (3.13) because both the delta function and its symmetric image contribute. From (2.16), in two dimensions the analogous quantity is

$$
\tilde{f}_{\mathrm{s}}(k)=\frac{2 \sqrt{2 \pi} C}{e v} \sqrt{\frac{\omega}{v_{\mathrm{F}}|k|}} .
$$

\subsection{Solution of the image source approximation}

It is instructive to compare the solution of the 'semiclassical' RPA equation, equation (2.14), with that of the image source approximation, equation (2.5). This is difficult in the twodimensional case, but quite straightforward in the case of a flat metallic surface in three dimensions. The charge density is still assumed to be a function of $z$ alone, and is related to the effective potential $\phi(z)$ by Poisson's equation:

$$
\frac{1}{e} \frac{\mathrm{d}^{2} \phi}{\mathrm{d} z^{2}}=\frac{\rho(z)}{\epsilon_{0}}
$$

so the charge density in (3.5) may be replaced by a term proportional to the second derivative of $\phi(z)$. (This simplification is not possible in two dimensions, because in that case $\rho$ also depends upon the second derivative with respect to the coordinate perpendicular to the conducting plane.) The resulting equation can be expressed in a scaled form analogous to (3.8), and is then transformed into an equation for the symmetrized potential, corresponding to (3.10). Here it is necessary to note that the symmetrized potential can have a discontinuity in slope at $z=0$, without the charge density having a singularity there. The electric field approaches a constant value as $z \rightarrow \infty$, and provided that $\omega \ll \omega_{\mathrm{p}}$, this field is much smaller than the externally applied field. If the internal field is neglected, the potential $\phi(z)$ may be assumed to approach zero as $z \rightarrow \infty$, and as $z \rightarrow 0_{+}$the slope $\mathrm{d} \phi / \mathrm{d} z$ approaches $-e q / \epsilon_{0}$, where $q$ is the integral of $\rho(z)$, i.e. the total charge per unit area bound to the surface. The symmetrized potential therefore has a discontinuity of slope equal to $-2 q e / \epsilon_{0}$ at $z=0$. The scaled potential $\psi_{\mathrm{s}}(x)$ satisfies

$$
\frac{\epsilon_{0}}{e^{2} v \Lambda^{2}} \frac{\mathrm{d}^{2} \psi_{\mathrm{s}}}{\mathrm{d} x^{2}}=\psi_{\mathrm{s}}(x)+\int_{-\infty}^{\infty} \mathrm{d} x^{\prime} G\left(x-x^{\prime}\right) \psi_{\mathrm{s}}\left(x^{\prime}\right)-\frac{2 q}{e v \Lambda} \delta(x) .
$$

This version of the RPA equation can also be solved directly by a Fourier transform approach. Noting that for ballistic electron motion in three dimensions $\epsilon_{0} / e^{2} v \Lambda^{2}=\omega^{2} / 3 \omega_{\mathrm{p}}^{2}$, the Fourier 
transform of the image source approximation solution is

$$
\tilde{\psi}_{\mathrm{s}}(k)=\frac{2 q \omega}{e \nu v_{\mathrm{F}}} \frac{1}{1+g_{3}(k)+\omega^{2} k^{2} / 3 \omega_{\mathrm{p}}^{2}} .
$$

This solution is indeed very close to the semiclassical RPA solution when $\omega \ll \omega_{\text {p }}$.

It should be emphasized that these forms for the effective potential are applicable only when the scale length $\Lambda$ is small compared to the characteristic dimension $a$ of the system. In the low-frequency limit, $\omega \ll \omega_{c}$, the scale size $\Lambda$ exceeds the size of the particle. In this limit a Thomas-Fermi approximation should be used for the effective potential. The form for the Fourier transform of the effective potential is then obtained from (3.11) or (3.17) by replacing the function $g_{3}(k)$ by zero.

\subsection{Components of the effective surface potential}

Reference [4], which considered the case where electron motion is diffusive, suggested writing the effective potential as the sum of two terms, namely a 'static' potential, which is given by the Thomas-Fermi approximation, and a 'dynamic' potential, which is proportional to frequency, and which is required to move the static polarization into place when the external electric field changes. Reference [2] showed that an additional component must be present in the ballistic case. It is interesting to see how these components are represented in equations (3.11), (3.13), and (3.17). The discussion will be restricted to the three-dimensional case.

Thomas-Fermi theory predicts that the potential is proportional to the charge density: $\phi(z)=\rho(z) / e v$. This is equivalent to setting $g(k)=0$ in (3.11) or (3.17). The resulting potential will be termed the 'static' potential, $\phi_{\text {stat }}(z)$. In the case of the semiclassical RPA method, it is simply a delta function localized at the surface, and in the case of the image source approximation it decays rapidly as a function of distance from the boundary, with decay length $\lambda_{\mathrm{s}}=\sqrt{\epsilon_{0} / e^{2} v}$.

In the vicinity of a surface which accumulates a polarization charge $q$, the internal electric field has magnitude $\mathcal{E}_{\text {int }}=\mathrm{i} \omega q / \sigma(\omega)$ where $\sigma(\omega)$ is the bulk conductivity. Reference [2] showed that this expression for the internal field is also applicable in the ballistic case, when the distance from the boundary is greater than $\Lambda=v_{\mathrm{F}} / \omega$. In the ballistic case, the bulk conductivity is $\sigma(\omega)=N e^{2} / \mathrm{i} m \omega$, where $N$ is the density of conduction electrons, and in the diffusive case $\sigma=e^{2} v D$ for frequencies small compared to the collision rate. There is therefore a component of the symmetrized potential which is proportional to $|x|$. In the ballistic case, this component of the scaled potential is

$$
\psi_{\mathrm{dyn}}(x)=\frac{i e \omega q \Lambda}{\sigma(\omega)}|x|=-\frac{3 q \omega}{2 e v v_{\mathrm{F}}}|x| .
$$

The generalized Fourier transform of $|x|$ is $-2 / k^{2}$. Consistency with (3.18) therefore requires that the Fourier transform of $\psi_{\mathrm{s}}(x)$ approaches $3 q \omega / e v v_{\mathrm{F}} k^{2}$ as $k \rightarrow 0$. Using equation (2.11) to evaluate the limit of (3.11) or (3.17) as $k \rightarrow 0$ verifies this relation.

The full effective potential may be written as the sum of $\phi_{\text {stat }}(z), \phi_{\text {dyn }}(z)$, and an additional term 'surface' term $\phi_{\text {surf }}(z)$, which decays with a characteristic length scale $\Lambda=v_{\mathrm{F}} / \omega$. The three components of the effective potential are illustrated schematically in figure 1 . The surface potential for the three-dimensional ballistic case is

$$
\begin{aligned}
\psi_{\text {surf }}(x)=\frac{2 q \omega}{e \nu v_{\mathrm{F}}} & \int_{0}^{\infty} \mathrm{d} k \exp (\mathrm{i} k x) \\
& \times\left\{\left[1-\frac{1}{2 k} \log _{\mathrm{e}}\left|\frac{k+1}{k-1}\right|+\frac{\mathrm{i} \pi}{2 k} \Theta(k-1)+\frac{1}{3}\left(\frac{\omega}{\omega_{\mathrm{p}}}\right)^{2}\right]^{-1}-1+\frac{3}{k^{2}}\right\}
\end{aligned}
$$




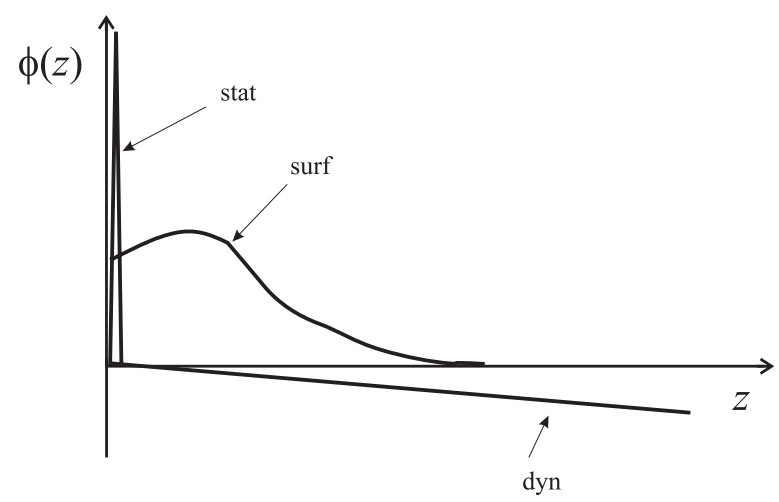

Figure 1. A schematic plot, illustrating the three components of the effective potential.

This expression diverges logarithmically as $x \rightarrow 0$ when $\omega / \omega_{\mathrm{p}}=0$.

In the case of diffusive electron motion it was shown in [8] that the effective potential is the sum of the static and dynamic components only. It is instructive to see how this conclusion is confirmed using the results of the present paper. In the diffusive case, it follows from (2.15) that the Fourier transform of the charge density is $\tilde{\rho}_{\mathrm{s}}(k)=2 q / e v$. Using (2.13) and (3.11), the Fourier transform of the effective potential is therefore

$$
\tilde{\phi}_{\mathrm{s}}(k)=\frac{2 q}{e v} \frac{1}{1+g_{\mathrm{D}}(\sqrt{D / \omega} k)}=\frac{2 q}{e v}\left[1-\frac{\mathrm{i} \omega}{D k^{2}}\right] .
$$

The first term in the final bracket Fourier transforms into a delta function, and therefore represents the static potential. The term proportional to $\omega / k^{2}$ Fourier transforms to a term proportional to $\omega|z|$, and represents the dynamic potential. The additional surface potential component is therefore absent in the diffusive case.

\section{Energy transferred on collision with surface}

The objective is to calculate the energy transferred to an electron from the externally applied electromagnetic field as it collides with the surface. From this point onwards, the discussion is specific to ballistic electron dynamics. The problem will first be treated classically, then the findings will be compared with the results of a quantum mechanical calculation.

\subsection{Classical treatment}

Classically, the energy transfer is determined by separate contributions from collisions with the surface of the particle, provided that the surface potential is localized at the surface of the particle. If the electron collides with the surface at time $t_{0}$, and is in the vicinity of the surface for a time $\Delta t$, the energy transferred at this collision is

$$
\delta E=\int_{t_{0}-\Delta t}^{t_{0}+\Delta t} \mathrm{~d} t \frac{\partial \phi}{\partial t}(\boldsymbol{r}(t), t)
$$

where $r(t)$ is the trajectory of the electron, and where the potential $\phi(r, t)$ is obtained by transforming the scaled potential $\psi(x)$ at frequency $\omega$ into a function of time:

$$
\phi(z, t)=\operatorname{Re}[\exp (\mathrm{i} \omega t) \psi(z(t) / \Lambda)] .
$$


For an electron incident at an angle $\theta$ from the normal to the surface, the distance from the surface at time $t$ is

$$
z(t)=v_{\mathrm{F}} \cos \theta\left|t-t_{0}\right| .
$$

Using the fact that $\psi(x)=\phi(x \Lambda)$ is symmetric about $x=0$, the energy transferred is therefore given by

$$
\delta E=-\omega \operatorname{Im}\left[\int_{-\infty}^{\infty} \mathrm{d} t \exp (\mathrm{i} \omega t) \psi\left(v_{\mathrm{F}} \cos \theta\left(t-t_{0}\right) / \Lambda\right)\right] .
$$

This may be expressed in terms of the Fourier transform of $\psi$ :

$$
\begin{gathered}
\delta E=\frac{-\Lambda}{v_{\mathrm{F}} \cos \theta} \operatorname{Im}\left[\exp \left(\mathrm{i} \omega t_{0}\right) \int_{-\infty}^{\infty} \mathrm{d} x \exp \left(\mathrm{i} x \omega \Lambda / v_{\mathrm{F}} \cos \theta\right) \psi(x)\right] \\
=\frac{-1}{\cos \theta} \operatorname{Im}\left[\exp \left(\mathrm{i} \omega t_{0}\right) \tilde{\psi}(1 / \cos \theta)\right] .
\end{gathered}
$$

Using (3.11), this result may be expressed in terms of the Fourier transform of $\psi(z)$ as follows:

$$
\delta E=\frac{-1}{\cos \theta} \operatorname{Im}\left[\exp \left(\mathrm{i} \omega t_{0}\right) \frac{\tilde{f}_{\mathrm{s}}(1 / \cos \theta)}{1+g(1 / \cos \theta)}\right] .
$$

Now consider the form of $\delta E$ for collision of a ballistic electron with the surface. In the two-dimensional case, combining (4.6), (2.9), and (3.14) gives

$$
\delta E=\frac{-C}{e \nu} \sqrt{\frac{2 \pi \omega}{v_{\mathrm{F}}}} \operatorname{Im}\left[\frac{\exp \left(\mathrm{i} \omega t_{0}\right)}{\sqrt{\cos \theta}(1+\mathrm{i} \cot \theta)}\right]
$$

which may be written as

$$
\delta E=\frac{C}{e v} \sqrt{\frac{2 \pi \omega}{v_{\mathrm{F}}}} \frac{\sin \theta}{\sqrt{\cos \theta}} \operatorname{Re}\left[\exp \left[\mathrm{i}\left(\omega t_{0}+\chi_{2}(\theta)\right)\right]\right]
$$

where $\chi_{2}(\theta)$ is a real-valued phase.

In the three-dimensional case the analogous expression, obtained using equations (2.10) and (3.12), is

$\delta E=\frac{2 q \omega}{e \nu v_{\mathrm{F}}} \operatorname{Im}\left[\frac{\exp \left(\mathrm{i} \omega t_{0}\right)}{\cos \theta\left(1+g_{3}(1 / \cos \theta)\right)}\right]=\frac{2 q \omega}{e \nu v_{\mathrm{F}}} S(\theta) \operatorname{Re}\left[\exp \left[\mathrm{i}\left(\omega t_{0}+\chi_{3}(\theta)\right)\right]\right]$

where

$S(\theta)=\frac{1}{\cos \theta}\left|\frac{1}{1-\frac{1}{2} \cos \theta\left[\log _{\mathrm{e}}(1+\cos \theta)-\log _{\mathrm{e}}(1-\cos \theta)\right]+(\pi / 2) \mathrm{i} \cos \theta}\right|$

and $\chi_{3}(\theta)$ is a phase. Both the two-dimensional and the three-dimensional expressions diverge at grazing incidence, where $\theta \rightarrow \pi / 2$. Physically, this can be interpreted in terms of the wavepacket spending a long time in contact with the barrier for a reflection at grazing incidence. A quantum mechanical treatment will take account of the fact that the wavefunction approaches zero at the surface. This removes the divergence.

\subsection{Quantum treatment}

Now consider how the expression (4.6) must be modified to take account of quantum mechanics. The approach will be to consider the collision of a wavepacket with the surface. The expectation value of the energy transferred will be calculated: this is

$$
\langle\delta E\rangle=\int_{-\infty}^{\infty} \mathrm{d} t \int_{0}^{\infty} \mathrm{d} z P(z, t) \frac{\partial \phi}{\partial t}(z, t)
$$


where $P(z, t)=|\Psi(z, t)|^{2}$ is the probability density for the electron to be at a distance $z$ from the surface. If the wavepacket can be chosen to be sufficiently well localized, the energy transfer can be assumed to be equal to this expectation value. On the other hand, if the length scale $L$ over which the wavepacket is localized is too large, then the expectation value will represent an average over the temporal variation of the potential, and $\langle\delta E\rangle$ will underestimate the magnitude of the energy transferred. The criterion is $L \ll v_{z} / \omega$, where $v_{z}=v_{\mathrm{F}} \cos \theta$ is the speed at which a Fermi-surface electron with angle of incidence $\theta$ approaches the surface. If the energy of the electron is to be well defined (and close to the Fermi level), then $k_{\mathrm{F}} L \ll 1$, where $k_{\mathrm{F}}$ is the Fermi wavevector. These two inequalities for $L$ are compatible provided that $\hbar \omega \ll E_{\mathrm{F}}$, which is assumed throughout.

The wavefunction of the wavepacket which collides with the surface at time $t_{0}=0$ may be written in the approximate form

$\Psi(z, t)=\exp \left(-\mathrm{i} E_{\mathrm{F}} t / \hbar\right)\left[\exp \left(\mathrm{i} p_{z} z / \hbar\right) f\left(z-v_{z} t\right)-\exp \left(-\mathrm{i} p_{z} z / \hbar\right) f\left(z+v_{z} t\right)\right]$

where $f(x)$ is a symmetric function, which decays rapidly when $|x| \gg L$. Dispersion of the wavepacket is unimportant, and is ignored in writing (4.12). The function $f$ is normalized, and its autoconvolution $F$ is required:

$$
F(x)=\int_{-\infty}^{\infty} \mathrm{d} x^{\prime} f\left(x-x^{\prime}\right) f\left(x^{\prime}\right) \quad F(0)=1 .
$$

Assuming that $\delta E=\langle\delta E\rangle$, and substituting (4.12) into (4.11) gives

$$
\begin{aligned}
\delta E=\frac{1}{2} \int_{-\infty}^{\infty} \mathrm{d} z \int_{-\infty}^{\infty} \mathrm{d} t \frac{\partial \phi}{\partial t}(z, t) \\
\quad \times\left[f^{2}\left(z-v_{z} t\right)+f^{2}\left(z+v_{z} t\right)-2 \cos \left(2 p_{z} z / \hbar\right) f\left(z-v_{z} t\right) f\left(z+v_{z} t\right)\right] .
\end{aligned}
$$

The integration over time will be performed first. It has been assumed that the envelope function varies much more rapidly than the variation in time of the potential $\phi(z, t)$, although the potential may vary rapidly as a function of $z$. Using (4.13), the energy transfer may therefore be approximated as follows:

$\delta E=\frac{1}{2 v_{z}} \int_{-\infty}^{\infty} \mathrm{d} z \frac{\partial \phi}{\partial t}\left(z, z / v_{z}\right)+\frac{\partial \phi}{\partial t}\left(z,-z / v_{z}\right)-2 \cos \left(2 p_{z} z / \hbar\right) F(2 z) \frac{\partial \phi}{\partial t}(z, 0)$.

If the potential varies sinusoidally in time, such that $\phi(z, t)=\operatorname{Re}[\exp (\mathrm{i} \omega t) \phi(z)]$, then (4.15) becomes

$\delta E=\frac{-\omega}{v_{\mathrm{F}} \cos \theta} \operatorname{Im}\left[\int_{-\infty}^{\infty} \mathrm{d} z \exp \left(\mathrm{i} \omega z / v_{\mathrm{F}} \cos \theta\right) \phi(z)-F(2 z) \phi(z) \cos \left(2 m v_{\mathrm{F}} z \cos \theta / \hbar\right)\right]$.

The first term of (4.16) is a Fourier transform of $\phi(z)$. Comparison with (3.17) shows that this takes the form

$$
\tilde{\phi}(k)=\frac{2 q}{e v}\left[\frac{1}{1+g_{3}\left(k v_{\mathrm{F}} / \omega\right)+\epsilon_{0} k^{2} / e^{2} v}\right] .
$$

The second term of (4.16) is the Fourier transform of the product $F(2 z) \phi(z)$ evaluated at $k=2 m v_{\mathrm{F}} \cos \theta / \hbar$. This may be obtained by convolution of (4.17) with the Fourier transform of $F(2 z)$. The function (4.17) has support $\sqrt{e^{2} v / \epsilon_{0}}=1 / \lambda_{\mathrm{s}}$, where $\lambda_{\mathrm{s}}$ is the Thomas-Fermi screening length, and has a structure close to $k=0$ associated with the function $g$, with a narrower support, $\omega / v_{\mathrm{F}}=1 / \Lambda$. The assumptions concerning the support $L$ of $F(z)$ imply that $\Lambda \gg L \gg \lambda_{\mathrm{s}}$. When calculating the Fourier transform of $\phi(z) F(2 z)$ using the convolution theorem, the structure in $\tilde{\phi}(k)$ associated with the function $g$ is suppressed, because the support 
of $\tilde{F}(k)$ is broader. The function $\tilde{\phi}(k)$ remains unchanged in other respects, because the support of $\tilde{F}(k)$ is narrower than the overall support of $\tilde{\phi}(k)$. The quantum mechanical expression for the energy transferred, calculated using the image source approximation for the effective potential, can therefore be approximated as follows:

$\delta E=\frac{-2 q \omega}{e \nu v_{\mathrm{F}} \cos \theta} \operatorname{Im}\left[\frac{1}{1+g_{3}\left(k v_{\mathrm{F}} / \omega\right)}-\frac{1}{1+4 \epsilon_{0} m^{2} v_{\mathrm{F}}^{2} \cos ^{2} \theta / \nu e^{2} \hbar^{2}}\right]$.

This simplifies to

$$
\delta E=\frac{2 q \omega}{e \nu v_{\mathrm{F}}} \operatorname{Im}\left[\frac{\cos \theta-\Gamma g_{3}(1 / \cos \theta) / \cos \theta}{\left(\Gamma+\cos ^{2} \theta\right)\left(1+g_{3}(1 / \cos \theta)\right)} \exp \left(\mathrm{i} \omega t_{0}\right)\right]
$$

where the time $t_{0}$ of collision with the surface has been inserted, and where

$$
\Gamma=\frac{e^{2} v \hbar^{2}}{4 \epsilon_{0} m^{2} v_{\mathrm{F}}^{2}} .
$$

The constant $\Gamma$ was introduced in [6]. It may be expressed in the alternative forms

$$
\Gamma=\frac{2 \lambda_{\mathrm{F}}}{\pi a_{0}}=\frac{2^{5 / 3}}{3^{2 / 3} \pi^{4 / 3}} \frac{r_{\mathrm{s}}}{a_{0}}
$$

where $a_{0}$ is the effective Bohr radius, and $r_{\mathrm{s}}$ is the radius of a sphere containing a single electron. Equation (4.19) will also be written in the form

$$
\delta E=\frac{2 q \omega}{e \nu v_{\mathrm{F}}} S(\theta) \cos \left[\omega t_{0}+\chi_{3}(\theta)\right]
$$

where

$$
S(\theta)=\left|\frac{\cos \theta-\Gamma g_{3}(1 / \cos \theta) / \cos \theta}{\left(\Gamma+\cos ^{2} \theta\right)\left(1+g_{3}(1 / \cos \theta)\right)}\right| .
$$

The formulae above should reduce to the Thomas-Fermi theory when the function $g_{3}$ is replaced by zero, and (4.20) does indeed reduce to equation (3.25) of reference [6] upon making this substitution.

The divergence of (4.10) at glancing incidence is absent in this more sophisticated quantum mechanical treatment, because the wavefunction of the electron vanishes at the surface, and the electron is therefore unable to be influenced by the potential there. Equation (4.19) approaches the prediction from (4.9) and (4.10) in the limit $\Gamma \rightarrow 0$ for all values of $\theta$ except $\pi / 2$, because as $\Gamma \rightarrow 0$ the Fermi wavelength becomes small compared to the Thomas-Fermi screening length, and the electron is able to penetrate closer to the surface.

In figure 2 the energy transferred to a reflected electron is plotted as a function of the angle of incidence $\theta$, for two different values of $\Gamma$, and for the semiclassical RPA, which corresponds to the limit $\Gamma \rightarrow 0$.

\section{Rate of absorption of energy}

\subsection{General considerations}

The absorption coefficient of a suspension of small particles is determined by the rate at which an individual particle absorbs energy. A semiclassical approach developed in earlier papers $[6,7,2]$ shows how the rate of absorption of energy by the electron gas may be expressed in terms of the variance of the change of energy of a single electron. For non-interacting fermions, the rate of change of the total energy $E_{\mathrm{T}}$ of the electron gas may be written as

$$
\frac{\mathrm{d} E_{\mathrm{T}}}{\mathrm{d} t}=V \nu D_{E}
$$




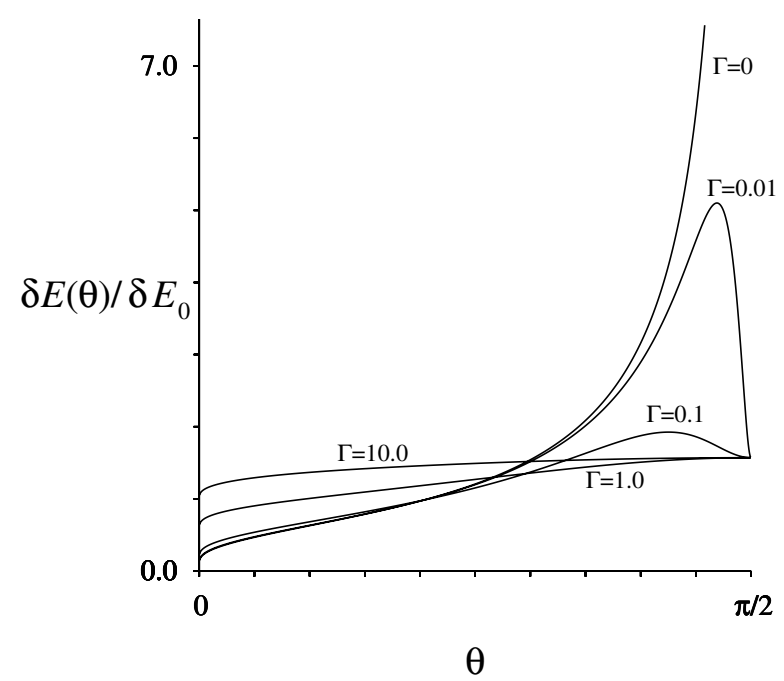

Figure 2. The energy $\delta E$ transferred to an electron rebounding from the surface, as a function of angle of incidence $\theta$. The scale energy is $\delta E_{0}=2 q \omega / e \nu v_{\mathrm{F}}$. This function depends upon the material-dependent parameter $\Gamma \propto r_{\mathrm{s}} / a_{0}$.

where $V$ is the volume of the particle, and $D_{E}$ is a diffusion constant for single-electron energies. The diffusion constant is defined by writing

$$
\left\langle\Delta E^{2}(t)\right\rangle=2 D_{E} t
$$

where $\Delta E(t)$ is the energy transferred to an electron after time $t$.

In the context of this paper $\Delta E(t)$ is the sum of the energy $\delta E_{j}$ transferred on collisions of the electron with the surface:

$$
\Delta E(t)=\sum_{j} \delta E_{j}
$$

where the sum runs over all collisions between times 0 and $t$. The variance in (5.2) is defined in terms of a phase-space average for electrons at the Fermi energy:

$\left\langle\Delta E^{2}(t)\right\rangle=\int \mathrm{d} \alpha \Delta E^{2}(\alpha) \delta\left(H(\alpha)-E_{\mathrm{F}}\right) / \int \mathrm{d} \alpha \delta\left(H(\alpha)-E_{\mathrm{F}}\right)$

where $\alpha=(\boldsymbol{q}, \boldsymbol{p})$ are phase-space coordinates of an electron, and $\Delta E(\alpha)$ is the energy transferred to an electron which is initially at $\alpha$.

In the ergodic case,

$$
\left\langle\Delta E^{2}(t)\right\rangle=R\left\langle\delta E^{2}\right\rangle t
$$

where $R$ is the rate of collision of particles with the boundary. The total distance travelled by the electron in time $t$ is $v_{\mathrm{F}} t=N\langle L\rangle$, where $\langle L\rangle$ is the mean distance travelled between each of the $N$ collisions. The rate of collisions is therefore

$$
R=\frac{v_{\mathrm{F}}}{\langle L\rangle} \text {. }
$$

\subsection{Absorption by conducting discs}

The simpler case in which to evaluate the phase-space average (5.4) is for two-dimensional discs, and this will be discussed in some detail to illustrate the approach. The calculation must 
be confined to the simpler semiclassical RPA approximation, because the more precise image source approximation has not been calculated in the two-dimensional case. The integrable case will be considered first, followed by the ergodic case (which applies when the surface of the disc is rough).

For the case of integrable electron motion in a disc, the angular momentum $J$ is a conserved quantity, and the coordinates $\left(E, J, t_{0}, \varphi_{0}\right)$ are a canonical set, where $E$ is the energy, $t_{0}$ the time since the most recent collision, and $\varphi_{0}$ is the polar angle of the most recent collision [7]. The angular momentum and the period $\tau$ between collisions are both related to the angle of incidence, $\theta$ :

$$
J=m a v_{\mathrm{F}} \sin \theta \quad \tau=\frac{2 a}{v_{\mathrm{F}}} \cos \theta
$$

where $a$ is the radius of the disc. Also, the charge density induced on a disc by an electric field $\mathcal{E}$ in the plane of the disc is

$$
\rho(r, \varphi)=\frac{4 \epsilon_{0} \mathcal{E} r \cos \varphi}{\pi \sqrt{a^{2}-r^{2}}}
$$

where $\varphi$ measured from the axis of the electric field [3]. The coefficient $C$ occurring in (2.16) is therefore given by

$$
C(s)=\frac{2 \sqrt{2}}{\pi} \epsilon_{0} \mathcal{E} \sqrt{a} \cos \varphi
$$

where $s=a \varphi$ is the distance around the perimeter. In two dimensions, the density of states is

$$
v=\frac{m}{\pi \hbar^{2}} .
$$

From (4.8), the total energy transferred to a single electron is

$$
\Delta E(t)=\sum_{j} \delta E_{j}=\frac{\pi \hbar^{2}}{m e} \sqrt{\frac{2 \pi \omega}{v_{\mathrm{F}}}} \frac{\sin \theta}{\sqrt{\cos \theta}} \sum_{j} C\left(s_{j}\right) \cos \left[\omega t_{j}+\chi_{2}(\theta)\right]
$$

where $t_{j}=j \tau+t_{0}$ are the times of the collisions with the surface, and the sum runs over $N \sim t / \tau$ values of the index $j$. The sum in (5.11) is dominated by resonances satisfying (1.2). However, in [6] it was shown that when $\omega \gg \omega_{c}$ equation (5.11) can be approximated by assuming that the bounces are independent events. This correctly describes the average behaviour of the absorption, but not that of the resonances. The mean squared energy transfer at fixed angle of incidence $\theta$ is then estimated to be

$$
\begin{aligned}
\left.\left\langle\Delta E^{2}(t)\right\rangle\right|_{\theta} & =N \frac{16 \pi \hbar^{4} \epsilon^{2} \mathcal{E}^{2} a \omega}{m^{2} e^{2} v_{\mathrm{F}}} \frac{\sin ^{2} \theta}{\cos \theta}\left\langle\cos ^{2} \phi_{j}\right\rangle\left\langle\cos ^{2}\left(\omega t_{j}+\chi_{2}(\theta)\right)\right\rangle \\
& =\frac{2 \pi \hbar^{4} \epsilon_{0}^{2} \mathcal{E}^{2} \omega}{m^{2} e^{2}} \frac{\sin ^{2} \theta}{\cos ^{2} \theta} t \equiv A \frac{\sin ^{2} \theta}{\cos ^{2} \theta} t
\end{aligned}
$$

where the final equality defines $A$. Now consider how to calculate the phase-space average in (5.4). The average over $\varphi_{0}$ has already been performed. It remains to average over $J$ and $t_{0}$. The quantity being averaged is independent of $t_{0}$, so integration over $t_{0}$ gives a contribution $\tau$. The required average is therefore

$$
\begin{aligned}
\left\langle\Delta E^{2}\right\rangle=\left.\int \mathrm{d} J \tau\left\langle\Delta E^{2}\right\rangle\right|_{\theta} / \int \mathrm{d} J \tau \\
=\left.\int_{0}^{\pi / 2} \mathrm{~d} \theta \cos ^{2} \theta\left\langle\Delta E^{2}\right\rangle\right|_{\theta} / \int_{0}^{\pi / 2} \mathrm{~d} \theta \cos ^{2} \theta \\
=A t \int_{0}^{\pi / 2} \mathrm{~d} \theta \sin ^{2} \theta / \int_{0}^{\pi / 2} \mathrm{~d} \theta \cos ^{2} \theta=A t .
\end{aligned}
$$


In the integrable case, the rate of absorption of energy is $\mathrm{d} E_{\mathrm{T}} / \mathrm{d} t=\frac{1}{2} \pi a^{2} v A$. The final expression for the rate of absorption of energy in the integrable case is then

$$
\frac{\mathrm{d} E_{\mathrm{T}}}{\mathrm{d} t}=\frac{\pi \hbar^{2} \epsilon_{0}^{2} a^{2} \mathcal{E}^{2} \omega}{m e^{2}} .
$$

It is interesting to note that this expression is independent of the Fermi energy. It may be written in the form

$$
\frac{\mathrm{d} E_{\mathrm{T}}}{\mathrm{d} t}=\frac{(e a \mathcal{E})^{2}(\hbar \omega)}{16 \pi \hbar E_{\mathrm{R}}}
$$

where $E_{\mathrm{R}}=m e^{4} / 16 \pi^{2} \epsilon_{0}^{2} \hbar^{2}$ is the Rydberg energy, $(a e \mathcal{E})$ is a measure of the energy associated with displacement of an electron across the particle by the electric field, and $\hbar \omega$ is the photon energy. This result differs by a numerical factor from that obtained in [6] using the ThomasFermi approximation for the effective potential.

If the edge of the disc is rough, with the result that the motion is ergodic, the rate of absorption of energy is calculated from (5.5) and (5.6). Using (4.8), (5.9), and (5.10), the mean squared value of the energy transferred at a single collision is

$$
\left\langle\delta E^{2}\right\rangle=\frac{4 \pi \hbar^{4} \epsilon_{0}^{2} \mathcal{E}^{2} a \omega}{m^{2} e^{2} v_{\mathrm{F}}}\left\langle\frac{\sin ^{2} \theta}{\cos \theta}\right\rangle .
$$

The average over the angle of incidence is

$$
\begin{aligned}
\left\langle\frac{\sin ^{2} \theta}{\cos \theta}\right\rangle= & \int \mathrm{d} J \tau \sin ^{2} \theta / \cos \theta / \int \mathrm{d} J \tau \\
& =\int_{0}^{\pi / 2} \mathrm{~d} \theta \cos \theta \sin ^{2} \theta / \int_{0}^{\pi / 2} \mathrm{~d} \theta \cos ^{2} \theta=\frac{4}{3 \pi} .
\end{aligned}
$$

Also,

$$
\langle L\rangle=v_{\mathrm{F}} \int \mathrm{d} J \tau^{2} / \int \mathrm{d} J \tau=2 a \int_{0}^{\pi / 2} \mathrm{~d} \theta \cos ^{3} \theta / \int_{0}^{\pi / 2} \mathrm{~d} \theta \cos ^{2} \theta=\frac{16 a}{3 \pi} .
$$

In the ergodic case, the rate of absorption is

$$
\frac{\mathrm{d} E_{\mathrm{T}}}{\mathrm{d} t}=\frac{\pi \hbar^{2} \epsilon_{0}^{2} a^{2} \mathcal{E}^{2} \omega}{2 m e^{2}}
$$

This differs by a factor of $\frac{1}{2}$ from the integrable case, given by (5.14).

\subsection{Absorption by conducting spheres}

The calculation of the energy absorbed proceeds by analogy with that for conducting discs. The first step is to specify a convenient set of phase-space coordinates. In the case of ballistic and specularly reflected electrons moving in a spherical enclosure, angular momentum is a conserved quantity. The following variables can be used to specify the phase-space coordinates of the electron: its energy $E$, angular momentum vector $\boldsymbol{J}$, and two angle variables, $\varphi$ and $\varphi^{\prime}$. In [6], it was shown that the measure $\mathrm{d} \alpha$ for canonical coordinates is given by

$$
\mathrm{d} \alpha=\frac{\tau}{J} \mathrm{~d} E \mathrm{~d} \varphi \mathrm{d} \varphi^{\prime} \mathrm{d} \boldsymbol{J} .
$$

The energy transferred to an electron bouncing at the surface is given by equations (4.19) or (4.22). The charge density at the surface is that given by classical electrostatics, namely

$$
q=3 \epsilon_{0} \mathcal{E} \cos \chi
$$


where $\mathcal{E}$ is the amplitude of the external electric field, and $\chi$ is the polar angle of the point on the surface measured from the direction of the electric field [3].

The rate of absorption of energy is calculated using (5.1) and (5.2). In the case of integrable motion (specular reflection at the surface), the mean squared energy transferred to the electron $\left\langle\Delta E^{2}\right\rangle$ is obtained by first averaging $\delta E^{2}$ at fixed angular momentum $\boldsymbol{J}$, and then integrating with respect to angular momentum. The variance of $\delta E$ at fixed $\boldsymbol{J}$ is

$\left.\left\langle\delta E^{2}\right\rangle\right|_{J}=\frac{9 \omega^{2} \epsilon_{0}^{2} \mathcal{E}^{2}}{e^{2} \nu^{2} v_{\mathrm{F}}^{2}} \cos ^{2} \chi_{0} S^{2}(\theta) \equiv A \cos ^{2} \chi_{0} S^{2}(\theta)$

where $\chi_{0}$ is the angle between the angular momentum vector $\boldsymbol{J}$ and the direction of the external electric field, and the final equality defines $A$. The number of collisions per unit time is $1 / \tau$. Treating the collisions as if they are independent events gives

$$
\begin{aligned}
\left\langle\Delta^{2} E(t)\right\rangle= & A t \int \mathrm{d} \boldsymbol{J} \frac{\tau}{J} \frac{1}{\tau} \cos ^{2} \chi_{0} S^{2}(\theta) / \int \mathrm{d} \boldsymbol{J} \frac{\tau}{J} \\
& =A t \int \mathrm{d} J \int \mathrm{d} \chi_{0} 2 \pi J^{2} \sin \chi_{0} \frac{1}{J} S^{2}(\theta) \cos ^{2} \chi_{0} / \int \mathrm{d} J 4 \pi J^{2} \frac{\tau}{J} \\
& =\frac{3 \epsilon_{0}^{2} \omega^{2} \mathcal{E}^{2}}{2 e^{2} \nu^{2} a v_{\mathrm{F}}} \int_{0}^{\pi / 2} \mathrm{~d} \theta \cos \theta \sin \theta S^{2}(\theta) / \int_{0}^{\pi / 2} \mathrm{~d} \theta \cos ^{2} \theta \sin \theta .
\end{aligned}
$$

The rate of absorption of energy by the spherical particle is therefore

$$
\frac{\mathrm{d} E_{\mathrm{T}}}{\mathrm{d} t}=\frac{3 \pi \epsilon_{0}^{2} a^{2} \mathcal{E}^{2} \omega^{2}}{e^{2} \nu v_{\mathrm{F}}} \mathcal{F}(\Gamma)
$$

where

$$
\mathcal{F}(\Gamma)=\int_{1}^{\infty} \mathrm{d} x \frac{1}{x} \frac{\left|1-\Gamma x^{2} g(x)\right|^{2}}{\left(1+\Gamma x^{2}\right)^{2}|1+g(x)|^{2}} .
$$

The integral $\mathcal{F}(\Gamma)$ diverges logarithmically as $\Gamma \rightarrow 0$ : for small $\Gamma, \mathcal{F} \sim K-\frac{1}{2} \log _{\mathrm{e}}(\Gamma)$, where $K$ is a constant. In the limit $\Gamma \rightarrow \infty, \mathcal{F}$ approaches a finite limit. Some values of $\mathcal{F}(\Gamma)$ obtained by numerical integration are given in table 1. A clearer understanding of (5.24) is obtained by expressing it in terms of ratios of energies: two equivalent forms are

$$
\frac{\mathrm{d} E_{\mathrm{T}}}{\mathrm{d} t}=\frac{3 \pi}{32} \frac{(a e \mathcal{E})^{2}(\hbar \omega)^{2}}{\hbar E_{\mathrm{F}} E_{\mathrm{R}}} \mathcal{F}(\Gamma)=\frac{3 \pi^{3}}{4} \frac{(a e \mathcal{E})^{2}(\hbar \omega)^{2}}{\hbar E_{\mathrm{R}}^{2}} \Gamma^{2} \mathcal{F}(\Gamma)
$$

Table 1. Values of the functions $\mathcal{F}(\Gamma)$ and $\mathcal{G}(\Gamma)$, defined by (5.25) and (5.30), obtained by numerical integration.

\begin{tabular}{rrr}
\hline$\Gamma$ & $\mathcal{F}(\Gamma)$ & $\mathcal{G}(\Gamma)$ \\
\hline 0.001 & 2.679 & 0.730 \\
0.01 & 1.594 & 0.609 \\
0.1 & 0.807 & 0.419 \\
1.0 & 0.813 & 0.489 \\
10.0 & 1.091 & 0.705 \\
100.0 & 1.149 & 0.750 \\
1000.0 & 1.155 & 0.755 \\
\hline
\end{tabular}

In the case where the surface of the spherical particle is rough, the electron motion is ergodic, and the rate of absorption is calculated via the microcanonical average of $\delta E^{2}$, using (5.5) and (5.6). A complicating feature is that the charge density concentrates on prominences 
of a rough surface. In [6] a simplified model was discussed, in which a fraction $\eta$ of the surface comprises high plateaus, with the charge density is increased by a factor $1 / \eta$, and the remainder of the surface is uncharged. According to this model, $\delta E^{2}$ is increased by a factor of $1 / \eta^{2}$ for a fraction $\eta$ of collisions. The required average is

$$
\begin{aligned}
\left\langle\delta E^{2}\right\rangle=\frac{2 A}{\eta} & \int \mathrm{d} \boldsymbol{J} \frac{\tau}{J} \cos ^{2} \chi S^{2}(\theta) / \int \mathrm{d} \boldsymbol{J} \frac{\tau}{J} \\
& =\frac{2 A}{\eta} \int \mathrm{d} J \int \mathrm{d} \chi 2 \pi J^{2} \sin \chi \frac{\tau}{J} \cos ^{2} \chi S^{2}(\theta) / \int \mathrm{d} J 4 \pi J^{2} \frac{\tau}{J} \\
& =\frac{2 A}{\eta} \int_{0}^{\pi / 2} \mathrm{~d} \theta \cos ^{2} \theta \sin \theta S^{2}(\theta)
\end{aligned}
$$

where $A$ is the factor defined in (5.22). The average distance between bounces is

$$
\langle L\rangle=v_{\mathrm{F}}\langle\tau\rangle=\frac{3 a}{2} .
$$

The rate of absorption is then found to be

$$
\frac{\mathrm{d} E_{\mathrm{T}}}{\mathrm{d} t}=\frac{8 \pi \epsilon_{0}^{2} a^{2} \mathcal{E}^{2} \omega^{2}}{\eta e^{2} \nu v_{\mathrm{F}}} \mathcal{G}(\Gamma)
$$

where

$$
\mathcal{G}(\Gamma)=\int_{1}^{\infty} \mathrm{d} x \frac{1}{x^{2}} \frac{\left|1-\Gamma x^{2} g(x)\right|^{2}}{\left(1+\Gamma x^{2}\right)^{2} \mid\left(1+\left.g(x)\right|^{2}\right.}
$$

The function $\mathcal{G}(\Gamma)$ approaches finite limits as $\Gamma \rightarrow \pm \infty$. Values obtained by numerical integration are given in table 1 . The tabulation demonstrates that the factors $\mathcal{F}$ and $\mathcal{G}$ are only weakly dependent upon $\Gamma$. Values of $\Gamma$ for real metals are numbers of order unity.

\section{Concluding remarks}

The principal new results in this paper are the solutions of the simplified RPA equations for the effective potential $\phi(z)$ (section 3), and the calculation of the energy transferred to an electron rebounding from the surface (section 4). They improve upon the earlier analysis in [6] and [7], which used a Thomas-Fermi approximation for the effective potential, rather than the random-phase approximation (RPA). In section 5 these results were used to obtain an improved estimate for the absorption of radiation by small particles, at frequencies small compared to the plasma frequency.

It is desirable to consider the limitations of some of the approximations which have been employed. The RPA prescription itself is an uncontrolled approximation, but it is expected to work well at high electron densities (small $r_{\mathrm{s}} / a_{0}$, or equivalently at small values of the parameter $\Gamma$ defined in (4.21)).

The image source method simplifies the calculation of the polarizability in two ways. Firstly, it treats the surface as if it were flat, and ignores contributions arising from reflections at more distant parts of the surface. These contributions are assumed to have relatively small amplitude and to combine incoherently, but their effect is very hard to quantify (except for slabs or strips with flat parallel faces). A second, and more significant, defect of the image source approximation is that it ignores interference effects between the direct and reflected paths. These are most significant when the amplitudes for the two paths are comparable: this happens when either $\boldsymbol{r}$ or $\boldsymbol{r}^{\prime}$ is close to the surface, and results in the polarizability vanishing as either point approaches the surface. The polarizability will differ from (2.4) by interference terms which oscillate with a wavenumber comparable with the Fermi wavenumber, $k_{\mathrm{F}}$. 
The semiclassical RPA approximation scheme makes the further assumption that the charge density has its classical distribution. In three dimensions the semiclassical RPA potential was compared with the image source approximation. The former has a delta function at the boundary, whereas the latter has a finite slope at $z=0$, corresponding to a rapid initial decay with length scale $\lambda_{\mathrm{s}}=\sqrt{\epsilon_{0} / e^{2} v}$ equal to the Thomas-Fermi screening length.

The parameter $\Gamma$ which appears in the image source theory for the energy transfer on reflection is small when $r_{\mathrm{s}} / a_{0}$ is small. In this limit the ratio of the Thomas-Fermi screening length to the Fermi wavelength, $\lambda_{\mathrm{s}} / \lambda_{\mathrm{F}}$, is large. When $\lambda_{\mathrm{s}}$ is small compared to the Fermi wavelength, the approximations used here are expected to fail, because the polarizability approaches zero within $\lambda_{F}$ of the surface, whereas the charge is expected to accumulate with a layer of thickness $\lambda_{\mathrm{s}}$. These considerations indicate that the theory is more accurate when $\Gamma$ is small, which corresponds to the case of good metals with a high density of conduction electrons. It is even possible that the results might be asymptotic to the results of an exact implementation of the RPA equations in the limit $\Gamma \rightarrow 0$.

In summary, the results presented in this paper represent an approximation scheme which leads to analytical expressions for the effective potential $\phi(z)$, and the energy transferred to an electron rebounding from the surface $\delta E(\theta)$. The latter was used to estimate the rate of energy absorption by a small particle due to an oscillating electric field. The resulting expressions are the first satisfactory analytical estimates for the low-frequency electric dipole absorption coefficient in the case of ballistic electron motion. They justify an earlier calculation, from reference [7], indicating that the absorption coefficient is proportional to frequency in the twodimensional case. They are expected to be a good approximation to the full RPA equations when $\Gamma$ is small.

\section{References}

[1] Fetter A L and Walecka J D 1971 Quantum Theory of Many-Particle Systems (New York: McGraw-Hill)

[2] Wilkinson M and Mehlig B 2000 J. Phys.: Condens. Matter $1210481-98$

[3] Landau L D and Lifshitz E M 1958 Electrodynamics of Continuous Media (Landau and Lifshitz Course of Theoretical Physics vol 8) (Oxford: Pergamon)

[4] Mehlig B and Wilkinson M 1997 J. Phys.: Condens. Matter 9 3277-90

[5] Bertsch G F and Broglia R A 1994 Oscillations in Finite Quantum Systems (Cambridge: Cambridge University Press)

[6] Austin E J and Wilkinson M 1993 J. Phys.: Condens. Matter 5 8461-84

[7] Wilkinson M and Austin E J 1994 J. Phys.: Condens. Matter $64153-66$

[8] Wilkinson M and Mehlig B 1998 Eur. J. Phys. B 1 397-8 